\title{
Medical Record Documentation System in Ethiopia
}

\author{
Addisu Tadesse Sahile ${ }^{1 *}$, Mussie Wubshet Teka ${ }^{2}$, Mikias Ababu Teshome ${ }^{2}$ and Tilahun Desalegn \\ Jata $^{3}$ \\ ${ }^{1}$ Department of Public Health, Unity University, Ethiopia
}

${ }^{2}$ Department of Public Health, Universal Medical and Business College, Ethiopia

${ }^{3}$ Independent consultant and Researcher in Addis Ababa, Ethiopia

*Corresponding author: Addisu Tadesse Sahile, Department of Public Health, Unity University, Addis Ababa, Ethiopia.

To Cite This Article: Addisu Tadesse Sahile, Mussie Wubshet Teka, Mikias Ababu Teshome, Medical Record Documentation System in Ethiopia. 2020 - 10(4). AJBSR.MS.ID.001534. DOI: 10.34297/AJBSR.2020.10.001534.

Received: 眥 September 24, 2020; Published: 鴜 October 12, 2020

\section{Introduction}

A medical record refers to a collection of data on a patient including but not limited to history, statement of the current problem, diagnosis, and treatment procedures [1]. Medical record completeness is one of the key indicators for the quality of healthcare delivery service [2]. The quality of the medical recording system can have a profound effect on the decision-making capabilities of the policy makers [3].

Globally, more than $50 \%$ of electronic medical record projects failed before reaching their target [4], whilst numerous health facilities have tried to implement with a very low success rate [5] Medical record keeping is an integral part of good professional practice in the delivery of quality health care. Irrespective of the form of the records (paper or electronic) keeping the clinical record of good quality helps for better communication among professionals and also for the continuity of the care [6]. Though there is great variability in the formats of the clinical notes among different health care professionals, hospitals, or countries [6], medical record-keeping must be seen as a learning tool with its importance [1].

Medical records should be able to be used as a reimbursement and legal tool something that is becoming increasingly crucial in the litigation of the today's culture [7], that could be updated when necessary by the efforts of every professional (Physician, Nurses, Pharmacy, Physiotherapist, student, psychologists, and occupational therapists) [6]. Medical records are useful to assess the quality of the health care provision, investigation of incidents, patient complaints, and compensation [6,8]. It will also help to ensure that every health professionals will have the proper information, reduces the time loss through repetition of tests, and averts inappropriate diagnosis, and treatments. Additionally, medical record-keeping helps for; aiding to target on diagnostics and treatments without unnecessary repetition, improve time management, sharing of relevant information, aid in continuity of care, coordination of care, informed decision making, provision of informative evidence in a court of law, improving availability of data for risk assessment and cause analysis and audit capabilities [6].

The records of the patient could represent the formal records of the clinician formal record, which must be clear, accurate, legible, and scientifically written. That could include all relevant clinical findings, decisions made, agreed actions, the person making the actions and decisions, the information given to patients, any prescribed drugs, made investigations, treatments, and when and who made the recording [6,9-11]. One of the mechanisms to practice evidence-based decision making and facilitated communication among health professionals uniformly to a common type of information is through the upgrading of the medical documentation system [12]

However, stating that 'good clinical record keeping facilitates decision making based on appropriately recorded information [6], 'is common parlance in eyes of policymakers', the level of complete medical documentation in Ethiopia was lower. Even though low, there was variability in medical record documentation practice in Ethiopia concerning time, place, and institutions [13]. The type of method applied was believed to have an impact on the completeness of medical record documentation in Ethiopia. The completeness was relatively higher in paper-based documentation 
[14]. Nowadays, besides improving the healthcare quality through availing appropriate information, the medical record has become part of the legal document for the protection of patient's safety $[2,15]$.

\section{Conclusion}

As to the observation of the author, complete medical record documentation is an unquestionable issue of the world population, as well as to Ethiopia. Multi-sectors with facilitation by the government must work rigorously on strengthening of the complete documentation system in Ethiopia. This could be the focus of future researchers in the area.

\section{Conflicts of Interest}

The authors declare that there is no conflict of interest.

\section{References}

1. Raza M (2012) Good Medical Record Keeping. International Journal of Collaborative Research on Internal Medicine \& Public Health 4(5).

2. Helfand M, Freeman M (2008) Assessment and Management of Acute Pain in Adult Medical Inpatients: A Systematic Review.

3. Adeleke IT, Adekanye AO, Onawola KA, Okuku AG, Adefemi SA, et al. (2012) Data quality assessment in healthcare: a 365-day chart review of inpatients' health records at a Nigerian tertiary hospital. Journal of the American Medical Informatics Association. 19(6): 1039-1042.

4. Ward R (2013) The application of technology acceptance and diffusion of innovation models in healthcare informatics. health Policy and Technology 2(4): 222-228.
5. Hasanain RA, Cooper H (2014) Solutions to overcome technical and social barriers to electronic health records implementation in Saudi public and private hospitals. Journal of Health Informatics in Developing Countries 8(1).

6. Mathioudakis A, Rousalova I, Gagnat AA, Saad N, Hardavella G (2016) How to keep good clinical records. Breathe 12(4): 369-73.

7. Great B, Standing Medical Advisory C (1965) The standardisation of hospital medical records; report of the sub-committee. London.

8. Pullen I, Loudon J (2006) Improving standards in clinical recordkeeping. Advances in psychiatric treatment 12(4): 280-286.

9. Wardle JL, Sibbritt D, Broom A, Steel A, Adams J (2016) Is health practitioner regulation keeping pace with the changing practitioner and health-care landscape? An Australian perspective. Frontiers in public health 4: 91.

10. Gill D, Griffin A (2010) Good medical practice: what are we trying to say? Textual analysis using tag clouds. Medical education 44(3): 316-322.

11. Samanta A, Samanta J, Gunn M (2003) Legal considerations of clinical guidelines: will NICE make a difference? Journal of the Royal Society of Medicine 96(3): 133-138.

12. (2012) Union IT. National eHealth Strategy.

13. Wong R, Bradley EH (2009) Developing patient registration and medical records management system in Ethiopia. International journal for quality in health care 21(4): 253-258.

14. Abiy R, Gashu K, Asemaw T, Mitiku M, Fekadie B, et al. (2018) A Comparison of Electronic Medical Record Data to Paper Records in Antiretroviral Therapy Clinic in Ethiopia: What is affecting the Quality of the Data? Online J Public Health Inform [Internet] 10(2): e212.

15. (2006) WHO WPR. Medical records manual: a guide for developing countries. Geneva: WHO Library Cataloguin in Publication Data. 